\title{
Study of the Flow Temperature and Ring Design Influence on the Response of a New Reduced-Size Calorimetric Cell for Nuclear Heating Quantification
}

\author{
A. Volte, C. Reynard-Carette, J. Brun, C. De Vita, M. Carette, T. Fiorido, A. Lyoussi, D. Fourmentel, \\ J-F. Villard and P. Guimbal
}

\begin{abstract}
This paper concerns experimental studies of different designs of a new compact calorimetric cell under laboratory conditions. This kind of cell is used for the measurement of the nuclear heating rate inside Material Testing Reactors thanks to differential calorimetry. The results, obtained by applying an operating protocol corresponding to a preliminary out-of-pile calibration step, are presented for three designs. The influence of the horizontal-fin design is shown on the calibration curve and the sensor sensitivity. The influence of the external fluid flow temperature is given for the quarter design. The different responses of the calorimetric cell are explained by taken into account a 1D analytical thermal model coupling thermal conductive and radiative transfers.
\end{abstract}

Index Terms-Calorimetry, New design, Calibration curve, Energy deposition rate, Nuclear fission.

\section{INTRODUCTION}

$\mathrm{C}$ ONTINOUS improvements in the nuclear energy field require research works. For instance, the enhancement of nuclear fuels or a better knowledge of material damages may benefit from feedbacks from existing nuclear power reactors, from qualified numerical models, and from experimental tests and qualification programs conducted in research infrastructures such as gamma or $\mathrm{X}$ ray sources, electron or ion accelerators, Zero Power Reactors and Material Testing Reactors (MTRs). To perform these kinds of programs, innovative and accurate instrumentation is needed to provide more and more accurate results and consequently to better understand complex phenomena occurring under harsh nuclear conditions.

This paper deals with research works on specific sensors called calorimeters dedicated to measuring online the energy deposition rate per units of mass induced by radiation interactions with matter in experimental channels of MTRs. The nuclear heating rate is a crucial parameter required during

A. Volte, C. Reynard-Carette, J. Brun, M. Carette, C. De Vita, and T. Fiorido are with Aix Marseille Univ, Université de Toulon, CNRS, IM2NP, Marseille, France (e-mail: adrien.volte@,im2np.fr, christelle.carette@univ-amu.fr, julie.brun@univ-amu.fr, michel.carette@univ-amu.fr, cedric.devita@im2np.fr, tomas.fiorido@im2np.fr). A. Lyoussi, D. Fourmentel, and J-F. Villard are with CEA, DEN, DER, Instrumentation Sensors and Dosimetry Laboratory, thermal and mechanical designs of experimental devices and after during result interpretation. Nuclear heating rate measurements in MTRs are usually performed directly by using single-cell calorimeters (including gamma thermometers) or differential calorimeters [1-4]. The measurement principle of these non-adiabatic calorimeters is almost similar and is based on temperature rise measurements by means of thermocouple (absolute temperatures or temperature differences). Then steady in-pile temperatures are associated to preliminary calibration curves (obtained under laboratory conditions or inside a reactor) to determine the nuclear heating rate. A single-cell calorimeter is composed of a jacket filled with conductive gas, a sample at its center, one or two thermocouples and sometimes a heating element. A differential calorimeter is made of two identical calorimetric cells at least (one containing a sample and the other kept empty to serve as a reference for energy deposition in the calorimetric-cell structure) with each cell instrumented by two thermocouples and a heating element dedicated to the in-pile or out-of-pile calibration.

Since 2009, Aix-Marseille University and French Alternative Energies and Atomic Energy Commission have been working together to improve calorimetric measurements thanks to differential calorimeter in particular and propose innovative sensors for the future Jules Horowitz Reactor (JHR) which is under construction at CEA Cadarache. The major challenges in these studies are to design sensors able to be operated up to a nuclear heating level of $20 \mathrm{~W} \cdot \mathrm{g}^{-1}$, and to reduce their size.

This paper focuses on experimental studies of three configurations of a new compact design of a differential calorimeter called CALORRE, and its tests under off-pile laboratory conditions without nuclear radiations.

The first part describes the experimental set-up previously developed [1,-3], the design of the three CALORRE calorimetric cells and the operating protocol applied to obtain the temporal responses of the sensors and to deduce their calibration curves.

Cadarache, F-13108, Saint Paul-lez-Durance, France (e-mail: abdallah..lyoussi@cea.fr, damien.fourmentel@cea.fr, jeanfrancoi.villard@cea.fr). P. Guimbal is with CEA, DEN, DER, Jules Horowitz Reactor Section, Cadarache, F-13108, Saint Paul-lez-Durance, France (e-mail: philippe.guimbal@cea.fr) 
The second part is dedicated to the presentation of the experimental results: the responses of the quarter-design configuration obtained for various thermal conditions (cooling fluid temperatures from $23^{\circ} \mathrm{C}$ to $63^{\circ} \mathrm{C}$ and electrical power up to $6 \mathrm{~W}$ ) and the calibration curves for the three designs at constant temperature equal to $33^{\circ} \mathrm{C}$. Then an analysis is performed thanks to a theoretical 1-direction thermal model based on an energy balance in the radial direction by considering thermal conductive exchanges in the horizontal fin, conductive exchanges in the gas layers located between the head and the vertical fin and the radiative exchanges between the outer surface of the head and the inner surface of the vertical fin.

The contribution of each heat transfer is discussed for all configurations to explain the different calibration curves.

\section{EXPERIMENTAL SET-UP AND OPERATING PROTOCOL}

\section{A. Experimental set-up}

The experimental set-up is composed of a thermostatic bath which contains a cylindrical jacket hosting a calorimetric cell, a power supply, a computer and an acquisition system linked to a high accuracy burster $0.10 \mathrm{hm}$ (cf. Fig.1.). The bath allows the control of the velocity for a large temperature range (from 23 to $63^{\circ} \mathrm{C}$ for our studies) of the cooling flow fluid (water in this case) that circulates in a parallel tube with a height of $400 \mathrm{~mm}$ and a diameter of $99 \mathrm{~mm}$. So, the cooling flow that operates in a vertical channel of an experimental research reactor is simulated by the vein of the bath. The Joule's effect generates with the power supply under non irradiation conditions inside each calorimetric cell imitates the nuclear heating rate occurring in materials inside the reactor. Temperatures of the calorimetric cell, the injected electrical current and the voltage are measured by means of an Agilent acquisition system coupled with 2 K-type thermocouples and a high accuracy burster respectively.

Three calorimetric cells were successively inserted inside the outer jacket and immersed into the vein of the bath to determine their response and establish their calibration curve. Each calorimetric cell is composed of a cylindrical head containing a sample and a heating element, a horizontal fin and a vertical fin. These three concentric parts are made of stainless steel (AISI 316L).

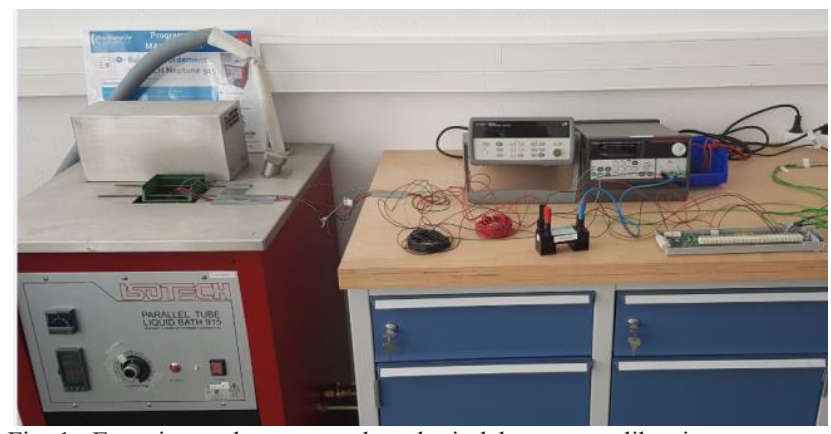

Fig. 1. Experimental set up used to obtain laboratory calibration curves

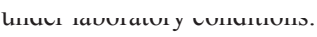

Two K-type thermocouples are inserted inside the horizontal fin: one close to the external surface of the head ( $\left.\mathrm{T}_{\text {hot }}\right)$ and one near the internal surface of the vertical fin at $180^{\circ}$ ( $\left.\mathrm{T}_{\text {cold }}\right)$. For each design a different geometry of the horizontal fin was tested. The heights of the calorimetric cells and of the horizontal fin are kept constant: $23.1 \mathrm{~mm}$ for the cell and $0.5 \mathrm{~mm}$ for the horizontal fin.

Figure 2 gives the picture of the quarter design of the CALORRE calorimetric cell and schemes of all tested designs. The quarter design has four metal sectors with an angle equal to $22.5^{\circ}$ and four empty sectors with an angle equal to $67.5^{\circ}$. The two other designs correspond to a full horizontal fin called full design and a half design with eight metal sectors and eight empty sectors with a same angle equal to $22.5^{\circ}$ respectively.

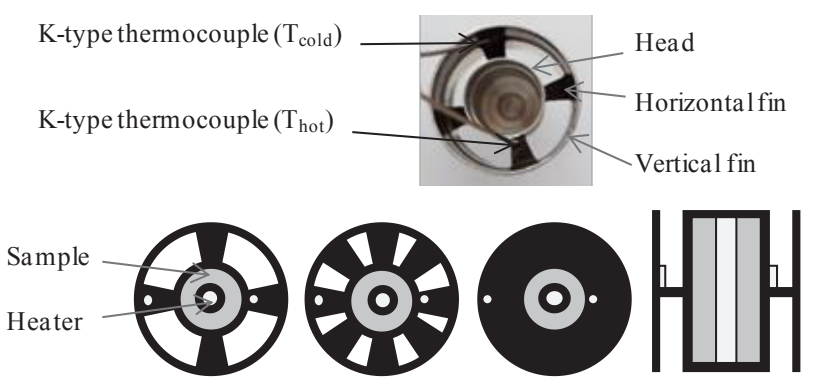

Fig. 2. Picture of CALORRE quarter design instrumented by two thermocouples and diagrams of the three designs (top-views of quarter, half and full designs and a general cross section).

\section{B. Operating protocol}

For each velocity and temperature, a specific electrical current is injected inside the heating element in such a way that the power range is from 0 to $6 \mathrm{~W}$. Temperatures and electrical power $(\mathrm{P})$ are measured as a function of the time. An increment of $0.5 \mathrm{~W}$ is carrying out at intervals of 30 minutes to have an acquisition time higher than the response time of the sensor in order to determine the mean temperature for a long steady state duration (cf. Fig.3.). For each applied electrical current the difference between the mean steady hot temperature and the mean steady cold temperature $(\Delta \mathrm{T})$ is calculated and plotted as a function of the injected electrical power. This kind of curve is called calibration curve (cf. Fig.4).

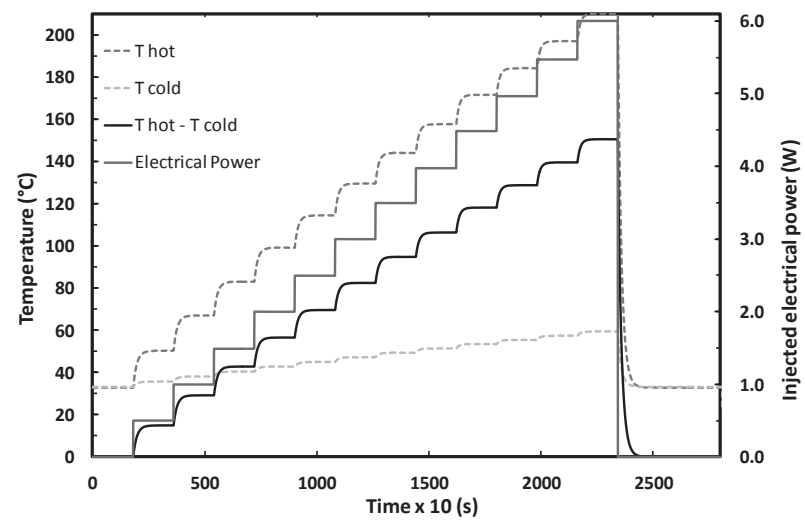

Fig. 3. Temperatures of CALORRE quarter design and injected electrical power versus time.

This protocol was followed for different temperatures (from $23^{\circ} \mathrm{C}$ to $63^{\circ} \mathrm{C}$ ) and velocities (from $\mathrm{Re}=609$ to $\mathrm{Re}=1607$ ) for the quarter design. 


\section{EXPERIMENTAL RESULTS}

Figure 4 presents the mean temperature difference versus the injected electrical power in the case of the quarter design of CALORRE for five fluid flow temperatures and a same fluid velocity corresponding to $\mathrm{Re}=1607$.

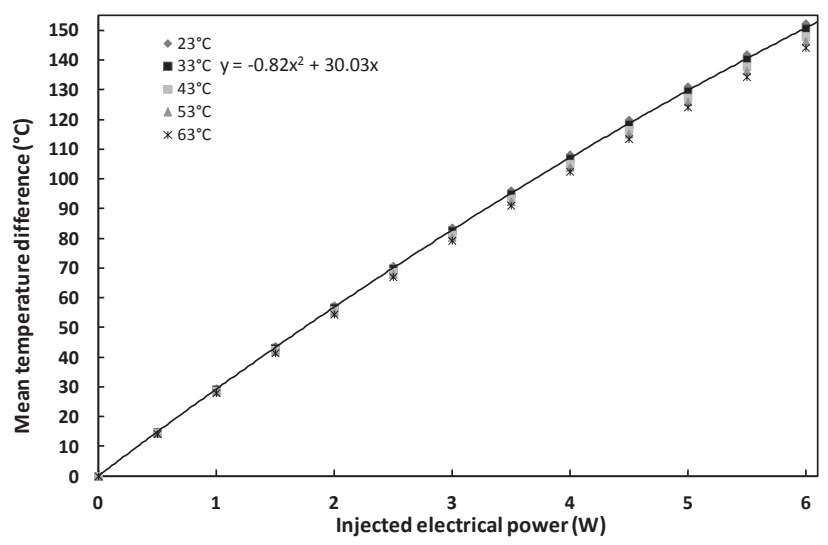

Fig. 4. CALORRE quarter design calibration curves obtained for several fluid flow temperatures.

First of all, whatever the fluid temperature the calibration curve is not linear. It corresponds to a second-order polynomial fit. Consequently, the sensitivity of this calorimetric cell decreases when the electrical power is increased. This behavior was already observed for CARMEN type calorimeter [1,3-4]. The flow temperature change leads to a variation of the calibration curve of the quarter-design cell. The mean temperature difference obtained for a same electrical power decreases with an increase of the fluid flow temperature.

Table 1 gives the calibration data obtained for the three designs of CALORRE for a same fluid temperature equal to $33^{\circ} \mathrm{C}$. These three designs have a second-order polynomial calibration curve too. More the horizontal fin contains empty sectors, higher is the sensitivity and the non-linearity. The sensitivity of the quarter design is higher than CALMOS type calorimeter sensitivity [2] and close to CARMEN type calorimeter sensitivity used during the MARIA campaign [5]. This is due to the different natures of material used for the calorimetric cell structures: stainless steel for CALORRE type calorimeter instead of aluminum for CALMOS and CARMEN type calorimeters.

TABLE I

Calibration Data For 3 CALORRE Designs AT $33^{\circ} \mathrm{C}$

\begin{tabular}{cccc}
\hline \hline $\begin{array}{c}\text { Sensor } \\
\text { design }\end{array}$ & $\begin{array}{c}\text { Equation of the } \\
\text { calibration curve }\end{array}$ & Sensitivity & $\begin{array}{c}\text { Sensitivity } \\
\text { in } 3 \mathrm{~W}\end{array}$ \\
& $\Delta \mathrm{T}$ in ${ }^{\circ} \mathrm{C}$ with $\mathrm{P}$ in $\mathrm{W}$ & ${ }^{\circ} \mathrm{C} . \mathrm{W}^{-1}$ & ${ }^{\circ} \mathrm{C} . \mathrm{W}^{-1}$ \\
\hline Full & $\Delta \mathrm{T}=-0.16^{*} \mathrm{P}^{2}+11.45^{*} \mathrm{P}$ & $-0.32 * \mathrm{P}+11.45$ & 10.49 \\
Half & $\Delta \mathrm{T}=-0.54 * \mathrm{P}^{2}+22.65 * \mathrm{P}$ & $-1.08 * \mathrm{P}+22.65$ & 19.41 \\
Quarter & $\Delta \mathrm{T}=-0.82 * \mathrm{P}^{2}+30.03 * \mathrm{P}$ & $-1.64 * \mathrm{P}+30.03$ & 25.11
\end{tabular}

The influence of the ring design and the flow temperature on the calibration curve can be explained by using a 1D thermal model. Indeed, due to the axisymmetric geometry of the calorimetric cell and by neglecting thermocouples, it is possible to apply a 1D thermal model by taking into account heat transfers only into the radial direction between the head and the vertical fin. This model is composed of three components.

The first term is due to the thermal conduction through the horizontal fin (into the metal and into the gas if this fin contains empty sectors). The power $\left(\mathrm{P}_{1}\right)$ induced by this heat exchange can be deduced from this formula:

$$
P_{1}=\frac{\left(T_{\text {hot }}-T_{\text {cold }}\right)}{\frac{\ln \left(\frac{r_{\text {head }}}{r_{\text {verticalfin }}}\right)}{H_{\text {horizontalfin }}\left(\text { netgas }+m \varphi \lambda_{\text {metal }}\right)}}
$$

with $\mathrm{r}_{\text {head }}$ the external radius of the head $(\mathrm{m}), \mathrm{r}_{\mathrm{verticalfin}}$ the internal

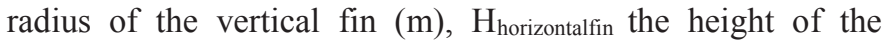
horizontal fin (m), $n$ the number of empty sectors, $m$ the number of metallic sectors, $\theta$ the angle of each empty sector (rad), $\varphi$ the angle of each metallic sector (rad), $\lambda_{\text {gas }}$ and $\lambda_{\text {metal }}$ the thermal conductivities $\left(\mathrm{W} \cdot \mathrm{m}^{-1} \cdot \mathrm{K}^{-1}\right)$ of the gas gap and the metal structure of the cell respectively estimated at the mean temperature $\left(\frac{T_{h o t}+T_{\text {cold }}}{2}\right)$ in $\mathrm{K}$.

The second term corresponds to the thermal conduction inside the gas gaps above and below the horizontal fin. The power evacuated through the gas gap $\left(\mathrm{P}_{2}\right)$ is given by this equation:

$P_{2}=\frac{2 \pi \lambda_{\text {gas }} H_{\text {gas }}\left(T_{\text {hot }}-T_{\text {cold }}\right)}{\ln \left(\frac{r_{\text {head }}}{r_{\text {verticalfin }}}\right)}$

with $\mathrm{H}_{\text {gas }}$ the total height of the gas gaps above and below the horizontal fin (m).

The last term relates to thermal radiative exchanges between the head and the vertical fin. The power can be estimated by using this expression:

$P_{3}=\frac{2 \pi \sigma H_{\text {gas }} r_{\text {head }}\left(T_{\text {hot }}{ }^{4}-T_{\text {cold }}{ }^{4}\right)}{\frac{1}{\varepsilon_{\text {head }}}+\frac{1-\varepsilon_{\text {verticalfin }}}{\varepsilon_{\text {verticalfin }}}\left(\frac{r_{\text {head }}}{r_{\text {verticalf in }}}\right)}$

with $\sigma$ the Stefan-Boltzmann constant $\left(\mathrm{W} \cdot \mathrm{m}^{-2} \cdot \mathrm{K}^{-4}\right), \varepsilon_{\text {verticalfin }}$ and $\varepsilon_{\text {head }}$ the emissivities of the internal surface of the vertical fin and the external surface of the head respectively.

Thanks to this 1D model, the contribution of each type of heat exchange can be deduced and used to explain the responses of the three designs. The results are given for an electrical power equal to $3 \mathrm{~W}$ for all designs in Figure 5 . The contribution of each thermal exchange depends on the horizontal fin design. In the case of the full design the main thermal contribution is the thermal conduction occurring into the ring. Consequently, the non-linearity of the calibration curve of the full design is mostly due to the variation of the thermal conductivity of the structure versus temperature. Then, the thermal conductive transfers through the ring are less important for the quarter design than those existing with the full design. But the thermal radiative transfers are enhanced in the case of the quarter design due to the presence of gas sectors which imply a greater thermal resistance of the horizontal fin and consequently an increasing in the sensor temperature. The new non-linear radiative component contributes also on the loss of the sensor linearity. According to the targeted nuclear heating level, this behavior should be taken into account to find a compromise to choose 
the suitable sensor configuration.

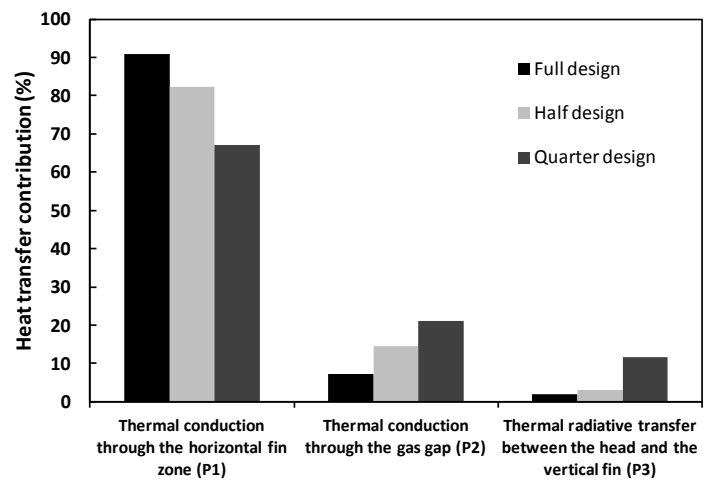

Fig. 5. Contribution of thermal conductive and radiative transfers for the three designs of CALORRE.

\section{CONCLUSIONS AND OUTLOOKS FOR Future STUdiES}

A new reduced-size calorimetric cell was studied under non irradiation conditions and for different ring configurations. The experimental results in laboratory show that the sensitivity of the compact sensor depends on the fluid temperature and can be tuned by changing the design of the horizontal fin. The sensitivity of the full design is equal to $10.49^{\circ} \mathrm{C} . \mathrm{W}^{-1}$ against $25.11^{\circ} \mathrm{C} . \mathrm{W}^{-1}$ for the quarter design for an injected electrical power of $3 \mathrm{~W}$. However, the ring change modifies the sensitivity and the non-linearity of the sensor response. The 1D thermal model allows the behavior interpretation. When the thermal resistance of the horizontal ring is increased, the sensitivity is higher but the non-linearity coefficient is greater too due to the variation of the thermal conductivity of the structure material versus the temperature and the increasing in the thermal radiative transfer.

This new compact calorimetric cell could be used for a higher nuclear heating rate such as $20 \mathrm{~W} \cdot \mathrm{g}^{-1}$ by choosing another structure material more conductive, and/or by reducing the size of the sensor to decrease the structure weight and consequently the undesired nuclear heating deposition on the structure leading to higher temperature field inside each cell, and/or by increasing the height of the horizontal fin. A new design will be studied by considering thermal property measurements versus temperature and by realizing $3 \mathrm{D}$ thermal numerical simulations with COMSOL Multiphysics with a complete differential calorimeter composed of two superposed calorimetric cells under laboratory conditions and then under irradiation conditions.

\section{ACKNOWLEDGMENT}

The IN-CORE program and MAHRI-BETHY programs are supported by FEDER, Conseil Régional PACA and Ville de Marseille.

\section{REFERENCES}

[1] J. Brun, M. Tarchalski, C. Reynard-Carette, K. Pytel, A. Lyoussi, J. Jagielski, D. Fourmentel, J-F. Villard and M. Carette, “ Numerical and Experimental Thermal Responses of Single-cell and Differential Calorimeters: Out-of-Pile Calibration to Irradiation Campaigns", IEEE Transactions on Nuclear Science, Vol. 63, N³, pp. 1630-1639, 2016.
[2] H. Carcreff, L. Salmon, J. Bubendorff and V. Lepeltier, "First In-Core Simultaneous Measurements of Nuclear Heating and Thermal Neutron Flux Obtained With the Innovative Mobile Calorimeter CALMOS Inside the OSIRIS Reactor", IEEE Transactions on Nuclear Science, Vol. 63, N5, pp. 2662-2670, 2016

[3] J. Brun, C. Reynard-Carette, A. Lyoussi, C. De Vita, M. Carette, M. Muraglia, D. Fourmentel, P. Guimbal, and J.-F. Villard, "Comparison of the Thermal Response of Two Calorimetric Cells Dedicated to Nuclear Heating Measurements during Calibration", IEEE Transactions on Nuclear Science, Vol. 61, N4, pp. 2254-2261, 2014.

[4] D. Fourmentel, C. Reynard-Carette, A. Lyoussi, J.F. Villard, J. Y. Malo, M. Carette, J. Brun, P. Guimbal and Y. Zerega, "Nuclear Heating Measurements in Material Testing Reactor: A Comparison Between a Differential Calorimeter and a Gamma Thermometer", IEEE Transactions on Nuclear Science, Vol. 60, $\mathrm{N}^{\circ} 1$, pp. 328-335, 2013

[5] J. Brun, C. Reynard-Carette, M. Carette, M. Tarchalski, K. Pytel, R. Prokopowicz, J. Jagielski, D. Fourmentel, L. Barbot, A. Lyoussi, J-F. Villard and P. Guimbal, "Irradiation Campaign in MARIA Reactor of an Innovative Calorimeter. Comparison of its Nuclear Heating Measurements with Two Kinds of Classical Sensors" presented at ANIMMA 2017, Liège, Belgium, 1923 June 2017 